\title{
Is It a Matter of Tempo? Music Tempo Effects on Food's Purchase Intentions and Perceived Taste: An Abstract
}

\author{
Felipe Pantoja and Adilson Borges
}

\begin{abstract}
Several sensory factors within the dining experience influence human eating patterns, food evaluations, perceptions, and consumption. A major, multifaceted, and complex variable of store atmospherics is background music. All musical compositions comprise five primary elements: timbre, mode, rhythm, tempo, and dynamics, and among those elements, tempo is considered to be the most likely to prompt listener responses. Tempo potentially operates on cognitive and emotional levels, both highly relevant to food consumption. Tempo manipulations have been shown to influence motor behaviors; that is, diners exposed to fast music tend to drink and eat more rapidly, but tempo effects on food perceptions and intentions have remained unexplored. Consequently, in this article we propose that a faster tempo will enhance taste perceptions and purchase intentions, and arousal and mood will mediate the effect in a sequential causal chain. In three studies, we show that faster rather than slower music tempo enhances food's perceived taste and purchase intentions and propose the aforementioned sequential affective route as the underlying mechanism. Finally, we add to the relatively scarce literature that focuses on auditory contributions to the sense of taste by showing that faster music tempo enhances taste perceptions and purchase intentions through changes in affective states. We also add to the literature on music tempo effects by showing that the temporal element alone positively influences consumers in the food context domain. Lastly, from a managerial standpoint, marketers should consider using faster musical excerpts for the background music in restaurants to trigger better customer responses. Store managers are advised to apply our results in real settings by modifying their background music.
\end{abstract}

\footnotetext{
F. Pantoja $(\bowtie)$

IÉSEG School of Management, Paris, France

e-mail: f.pantoja@ieseg.fr
}

\author{
A. Borges \\ NEOMA Business School, Reims, France \\ e-mail: adilson.borges@neoma-bs.fr
}

